\title{
Wireless channel estimation in OFDM systems based on collaborative filtering techniques
}

\author{
Velimir Švedek ${ }^{*}$, Adrian Satja Kurdija ${ }^{* *}, \check{Z ̆ e l j k o ~ I l i c ́ ~}^{* *}$
}

\begin{abstract}
In this paper, a new channel estimation algorithm in Orthogonal Frequency Division Multiplexing (OFDM) systems is proposed. The proposed algorithm is suitable for cases with low density of pilot sub-carriers, where standard interpolation methods (linear, second order and cubic spline interpolation) are inaccurate. The algorithm improves the interpolation methods by employing memory based collaborative filtering $(\mathrm{CF})$ techniques which are less sensitive to the number and location of the pilot subcarriers. CF algorithms are usually used in the context of recommender systems (e-commerce) for predictions of the unknown user-item ratings based on known values of similar users. The advantage of CF is the ability to efficiently produce quality predictions with highly sparse data. Computer simulations are used to verify the proposed channel estimation algorithm and demonstrate that the proposed algorithm improves predictive accuracy metrics, such as Root Mean Squared Error (RMSE), compared to usual estimation methods.
\end{abstract}

K e y w o r d s: OFDM, channel estimation, collaborative filtering, interpolation methods

\section{Introduction}

Over the last decade, the accelerated development of wireless broadband access technologies has been noted as a response to the increasing demand for higher quality services with heterogeneous demands on quality of service (QoS). An increasing number of different (dynamic) applications with different QoS parameters simultaneously demand different transmission data rates from the network, with supported latency ranges from a few milliseconds up to a few seconds, and a number of connections that can change to tens of thousands [1]. Orthogonal Frequency Division Multiplexing (OFDM), as one of the most widespread modulation techniques successfully deals with above-mentioned requirements. It is actively used in wireless broadband systems such as IEEE 802.16 (WiMAX), digital video broadcasting-terrestrial (DVB-T) and 3GPP (LTE, LTE-Advanced) [2]. OFDM, as a modulation technique where available frequency spectrum is divided into several orthogonal subcarriers, is characterized by simple implementation, high transmission data rates, high spectral efficiency and robustness against multipath propagation [3].

As the channel in wireless communication systems is time variant and frequency selective, efficient and accurate channel estimation is needed for a coherent detection of transmitted data, and it is therefore a challenging task. Channel estimation is aimed to estimate the time domain or the frequency domain response of the channel in order to increase the capacity of an OFDM system by improving the bit error rate (BER) performance. In OFDM, capacity of the system highly depends on the knowledge of channel characteristics (Channel State Information CSI) between transmitter and receiver and consequently, if channel distortion effect for each of the active subcarriers within an OFDM symbol is estimated accurately, undesirable effects on channel response can be compensated [4]. Channel estimation methods can be grouped into two categories: non-pilot aided and pilot aided (block and comb type) [5].

In OFDM based systems, pilot-aided comb type channel estimation usually involves some form of interpolation for estimation of channel states at non-pilot (data) subcarriers. Interpolation methods can have a strong influence on accuracy of channel estimation. Interpolation methods that are commonly used are linear interpolation, second order interpolation, and spline cubic interpolation [5]. Currently there is a great interest in channel estimation techniques and interpolation methods that are used in OFDM systems and thus have been studied by many researchers $[6-8]$. However, the performance of most of them is limited by either the number or the position of pilot subcarriers [5].

In this paper, we propose a new channel estimation algorithm which improves the commonly used interpolation methods by employing memory based collaborative filtering ( $\mathrm{CF}$ ) techniques, which are less sensitive to the number and location of the pilot subcarriers. Such channel estimation algorithms based on irregular and scarce pilot arrangements can be very useful in orthogonal frequency division multiple access (OFDMA) systems where different user channels need to be simultaneously determined at the base station which can induce an irregular overall pilot arrangement [9].

*Croatian Regulatory Authority for Network Industries, Zagreb, Croatia, velimir.svedek@hakom.hr, **Faculty of Electrical Engineering and Computing, University of Zagreb, Unska 3, Zagreb, Croatia, (adrian.kurdija, zeljko.ilic)@fer.hr 


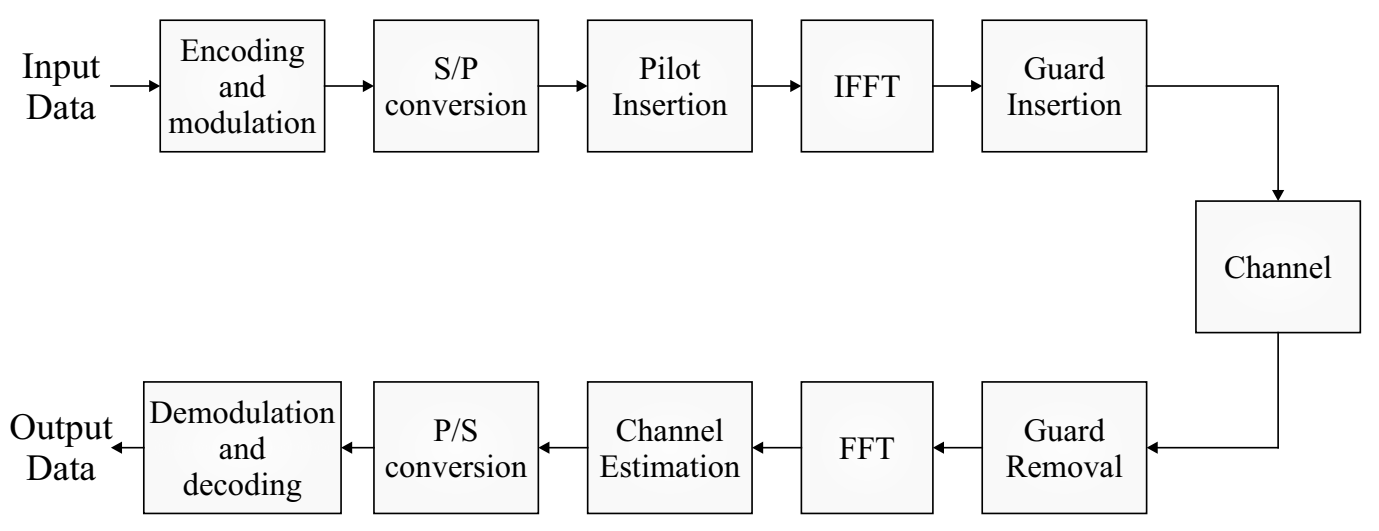

Fig. 1. Baseband OFDM system [5]

Frequency

\begin{tabular}{|lllllll|}
\hline & 4 & Block & \multicolumn{2}{c|}{ pilot data } \\
0 & 0 & 0 & 0 & 0 & \multicolumn{2}{c|}{0} \\
0 & 0 & 0 & 0 & 0 & 0 \\
0 & 0 & 0 & 0 & 0 & 0 \\
0 & 0 & 0 & 0 & 0 & 0 & 0 \\
0 & 0 & 0 & 0 & 0 & 0 & 0 \\
0 & 0 & 0 & 0 & 0 & 0 & 0 \\
0 & 0 & 0 & 0 & 0 & 0 & 0 \\
0 & 0 & 0 & 0 & 0 & 0 & 0 \\
0 & 0 & 0 & 0 & 0 & 0 & 0 \\
\hline
\end{tabular}

Time
Frequency

\begin{tabular}{|c|c|c|c|c|c|c|}
\hline$\bullet$ & 0 & 0 & 0 & 0 & 0 & $\gamma^{\text {pilot }}$ \\
\hline 0 & 0 & 0 & 0 & 0 & 0 & $o^{\text {data }}$ \\
\hline 0 & 0 & 0 & 0 & 0 & 0 & 0 \\
\hline 0 & 0 & 0 & 0 & 0 & 0 & 0 \\
\hline$\bullet$ & 0 & 0 & 0 & 0 & 0 & 0 \\
\hline 0 & 0 & 0 & 0 & 0 & 0 & 0 \\
\hline 0 & 0 & 0 & 0 & 0 & 0 & 0 \\
\hline 0 & 0 & 0 & 0 & 0 & 0 & 0 \\
\hline 0 & ○ & 0 & 0 & 0 & 0 & 0 \\
\hline
\end{tabular}

Time

Fig. 2. Major methods of pilot arrangement for OFDM channel estimations [14]

$\mathrm{CF}$ techniques in a recommender system are using knowledge about a set of users preferences to make predictions of other unknown user preferences. CF algorithms can deal with highly sparse data and the increasing number of users/items while at the same time making satisfactory recommendations in a short period of time. CF techniques can be divided into three categories: memory-based, model-based, and hybrid CF techniques [10]. Memory-based CF methods, which are using the entire or a sample of the user-item database to generate a prediction, are mostly deployed into e-commerce systems because of their easy implementation and high effectiveness [11]. If we assume that every user is a part of a group of people with similar interests, then by identifying "neighbours" of an active/new user, a prediction of related preferences on new items can be produced [12].

In our newly proposed channel estimation algorithm, we have modified CF logic for the users of a wireless network in a way that items correspond to the subcarriers of respective users. In a prediction of unknown channel states at non-pilot subcarriers, the assumption was made that channel states at subcarriers of similar (close) users are behaving similarly.

\section{Wireless channel estimation and interpolation techniques in OFDM systems}

OFDM is a type of a low complexity modulation technique that allows data transmission by radio waves and as such is widely used in radio transmission systems. In general, this modulation allows data transmission using many parallel narrow-band sub-streams. Each sub-stream is modulated on closely spaced orthogonal frequency subcarriers. Orthogonality of the subcarriers is crucial in OFDM for simultaneous transmission of data as there is no interference between adjacent subcarriers [13]. The block diagram of OFDM transmitter/receiver system is presented in Fig. 1. In OFDM systems, pilot symbols (known signals for the receiver and transmitter) are typically inserted into data streams, allowing the channel estimation within the receiver. The pilot symbols can be scattered in the time and/or frequency directions in OFDM frames.

Although the main part of an OFDM transmission is the FFT/IFFT operation, capacity of an OFDM system highly depends on CSI and, because of that, it is crucial to estimate the channel accurately, so that undesirable effects on channel response can be compensated [14]. 
Various techniques for channel estimation in OFDM systems have been proposed $[15,16]$. There are two categories: non-pilot aided, and pilot aided. The pilot aided techniques provide better results while sacrificing some part of available bandwidth for pilot data [15]. The pilot aided channel estimation techniques can be performed by either inserting pilot symbols with a specific period into all subcarriers of OFDM symbols (block type) or inserting pilot symbols into each OFDM symbol (comb type) [16], as shown in Fig. 2. Block type is better for slower fading channels while comb type is better for faster fading.

The estimation of the channel for block-type technique arrangement can be based on Least Square (LS) or Minimum Mean-Square Error (MMSE) algorithms. LS and MMSE algorithms are used for obtaining channel information at pilot subcarriers [17].

In a comb-type technique, after obtaining the channel information at pilot subcarriers, the OFDM system receiver needs to use an efficient interpolation technique to estimate channel values at all non-pilot subcarriers $[18,19]$. High transmission rates and low BER in OFDM systems force the use of interpolation methods that simultaneously fulfil low complexity and high accuracy, as these requirements are crucial for the accuracy of channel estimation. In general, interpolation is used to estimate unknown data points between known data points. Popular one-dimensional interpolation methods like linear interpolation, second-order polynomial interpolation, and cubic spline interpolation, are often adopted in OFDM systems to accomplish the trade-off between complexity and accuracy $[5,6,20,21]$.

\subsection{Linear interpolation}

In linear interpolation, the frequency response of data subcarriers is considered as a straight line between two adjacent pilot subcarriers. Using linear interpolation, estimation at the frequency of a data subcarrier $k$ located between the $m^{\text {th }}$ and $(m+1)^{\text {th }}$ pilot subcarrier which are spaced by $L$ is given by [22]

$$
\begin{array}{r}
\widehat{H}(k)=\widehat{H}(m L+l)=\left(1-\frac{l}{L}\right) \widehat{H}_{p}(m)+\frac{1}{L} \widehat{H}_{p}(m+1) \\
=\widehat{H}_{p}(m)+\frac{l}{L}\left(\widehat{H}_{p}(m+1)-\widehat{H}_{p}(m)\right), \quad 0 \leq l<L
\end{array}
$$

where $\widehat{H}_{p}(m)$ is the value of the received pilot subcarrier at the frequency $m$.

Linear interpolation has a low computational complexity and gives best performance in models where there are enough pilot subcarriers and where the subcarriers are close to each other [23]. Although linear interpolation significantly degrades BER performance if the channel is changing fast between neighbouring pilot subcarriers [8] (which is a non-linear behaviour), it is still most frequently used in OFDM systems because of its easy implementation and inherent simplicity.

\subsection{Second order interpolation method}

As linear interpolation requires more pilot subcarriers for a better performance in highly frequency selective channels, usage of higher order polynomials can result in better estimation performance but at the expense of higher computational complexity [19]. Polynomials of higher order are better for highly time and frequency selective channels, otherwise use of them can degrade the performance, as the modelling uses noise to represent the channel [24]. Channel estimation by second order interpolation is given by [22]

$$
\begin{aligned}
\widehat{H}(k)= & \widehat{H}(m L+l) \\
& =c_{1} \widehat{H}_{p}(m-1)+c_{0} \widehat{H}_{p}(m)+c_{-1} \widehat{H}_{p}(m+1)
\end{aligned}
$$

with

$$
\begin{aligned}
& c_{1}=\alpha(\alpha+1) /(2), \quad c_{0}=-(\alpha-1)(\alpha+1), \\
& c_{-1}=\alpha(\alpha-1) / 2 \quad \text { and } \quad \alpha=\frac{l}{N},
\end{aligned}
$$

where, $N$ is the number of subcarriers in an OFDM symbol.

\subsection{Cubic spline interpolation}

Cubic spline curve, as a piecewise curve, can reflect the change trend of real curves more accurately by fitting a series of unique cubic polynomials between each of subcarriers [25]. To make estimation, values are obtained using the following equation [16]

$\widehat{H}(k)=\widehat{H}(m L+l)=\alpha_{1} \widehat{H}_{p}(m+1)+\alpha_{0} \widehat{H}_{p}(m)+$
$L \alpha_{1} \widehat{H}^{\prime} p(m+1)-L \alpha_{0} \widehat{H}^{\prime} p(m), m=0,1, \ldots, N_{p}-1$,

where $\widehat{H}_{p}^{\prime}(m)$ is the first order derivative of $\widehat{H}_{p}(m), N_{p}$ is the number of pilot symbols and

$$
\begin{aligned}
& \alpha_{1}=3 \frac{(L-l)^{2}}{L^{2}}-2 \frac{(L-l)^{3}}{L^{3}}, \\
& \alpha_{0}=3 \frac{l^{2}}{L^{2}}-2 \frac{l^{3}}{L^{3}} .
\end{aligned}
$$

\section{Collaborative filtering techniques}

Collaborative filtering ( $\mathrm{CF}$ ) techniques are among the most successful techniques for building recommender systems [12]. Recommender systems are based on learning systems and are used in e-commerce to recommend/predict useful items to new users from its databases of preferences for items by users. CF techniques use the known preferences of a group of users to make estimates of the unknown preferences for other users, with general assumption that if users $X$ and $Y$ rate some items similarly then they will also rate other items similarly [10]. To make satisfactory recommendations in a short time period, $\mathrm{CF}$ algorithms are required to manage highly sparse 


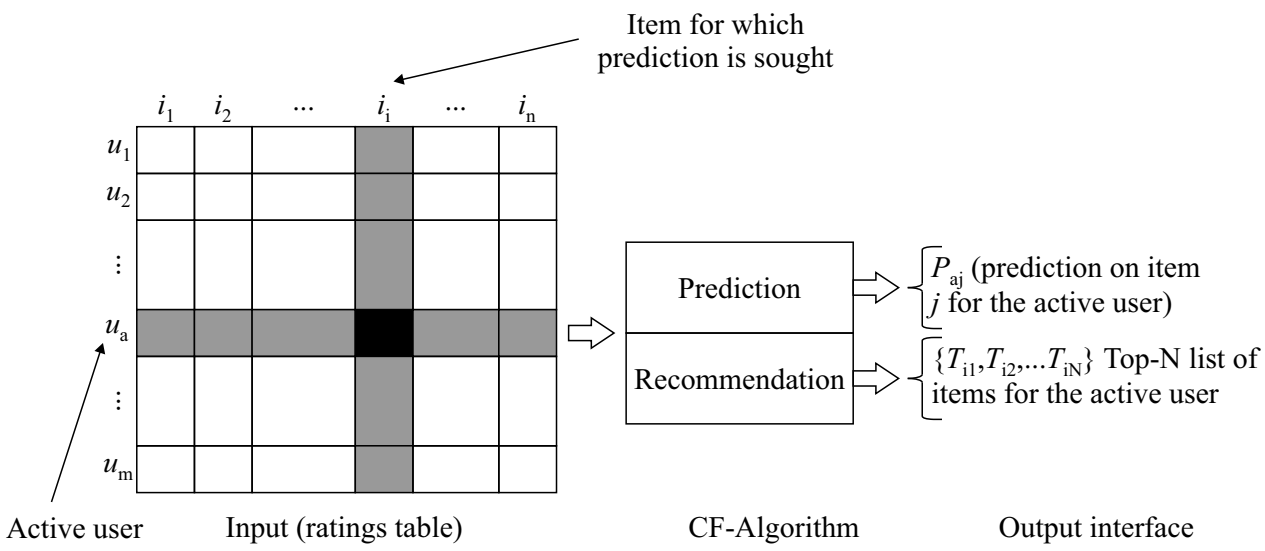

Fig. 3. Schema of the CF process [27]

data and to scale with increasing numbers of users and items.

Typically, in each CF scenario [26], there is a list of $\mathrm{m}$ users $U=\left\{u_{1}, u_{2}, \ldots, u_{m}\right\}$ and a list of $n$ items $I=\left\{i_{1}, i_{2}, \ldots, i_{n}\right\}$. Each user $u_{i}$ has associated list of items $I_{U_{i}}$ which the user has rated. User ratings are stored in a rating matrix $\boldsymbol{V}$ which is used for generating recommendations. Element $r_{u}$, i of $\boldsymbol{V}$ denotes the rating given by user $u$ to item $i$, while value 0 indicates that the item is not rated yet. Objective of the CF algorithm is to accurately predict the values of $\boldsymbol{V}$ in these cases as shown in Fig. 3.

Depending on how the data of the rating matrix are processed, CF algorithms can be divided in two main categories: memory-based and model-based [28]. The memory-based algorithms are broadly deployed into commercial systems because they are easy-to-implement and highly effective [12]. They are using the entire or a sample of the user-item database to generate predictions. With assumption that every user is a part of some group of users with similar interests, by identifying the "neighbours" of a new user a prediction of her/his ratings on new items can be produced. Model-based CF algorithms differ from memory-based by first developing and learning a model to find patterns of user ratings using the pure rating data. The most popular approaches for model-based $\mathrm{CF}$ algorithms are performed with machine learning models such as Bayesian Belief Net models and Clustering models [28]. In this paper we propose to employ a memory-based CF approach in a previously unused context, to address challenges of wireless channel estimation in OFDM systems, especially the sensitivity to the number and location of the pilot subcarriers.

\subsection{CF-based estimation algorithm}

Memory-based CF algorithms are using an entire rating matrix to make predictions. Commonly, they use neighbourhood-based statistical techniques to select users that are like the target user. Prediction to a user is then calculated from the ratings of these neighbours, taking into account the similarity or weight $w_{u, v}$ which reflects the ratings correlation between users $u$ and $v$, and taking the weighted sum (a simple weighted average) of all considered ratings for that item [29].

\subsubsection{Similarity computation}

To accurately compute the correlation-based similarity $w_{u, v}$ between two users $u$ and $v$ that have rated some common items, we first must isolate the co-rated cases (items rated by both $u$ and $v$ ). Then correlation-based similarity can be measured by computing the Pearson correlation, which is widely used in the CF research community, between users $u$ and $v$ [12]

$$
w_{u, v}=\frac{\sum_{i \in I}\left(r_{u, i}-\bar{r}_{u}\right)\left(r_{v, i}-\bar{r}_{v}\right)}{\sqrt{\sum_{i \in I}\left(r_{u, i}-\bar{r}_{u}\right)^{2}} \sqrt{\sum_{i \in I}\left(r_{v, i}-\bar{r}_{v}\right)^{2}}}
$$

where $I$ is the set of co-rated items, and $\bar{r}_{u}$ and $\bar{r}_{v}$ denote the average rating of the co-rated items by $u^{\text {th }}$ and $v^{\text {th }}$ user.

\subsubsection{Prediction computation}

The goal of a CF technique is to generate the prediction. Once we isolate the subset of nearest neighbours of the target user, the next step is to investigate the target users ratings and make a weighted aggregate of their ratings to make a prediction [12].

Prediction of the rating of user $\mathrm{v}$ on item $i$ is calculated according to the following equation [30]

$$
P_{v, i}=\bar{r}_{v}+\frac{\sum_{u \in U}\left(r_{u, i}-\bar{r}_{u}\right) w_{v, u}}{\sum_{u \in U}\left|w_{v, u}\right|}
$$

where $U$ is the set of nearest neighbours of $v, \bar{r}_{v}$ and $\bar{r}_{u}$ are the average ratings for user $v$ and user $u$ on all other rated items, and $w_{v, u}$ is the weight (similarity) between the user $v$ and user $u$. If a user is more like the target user, the estimation is going to be more accurate. 


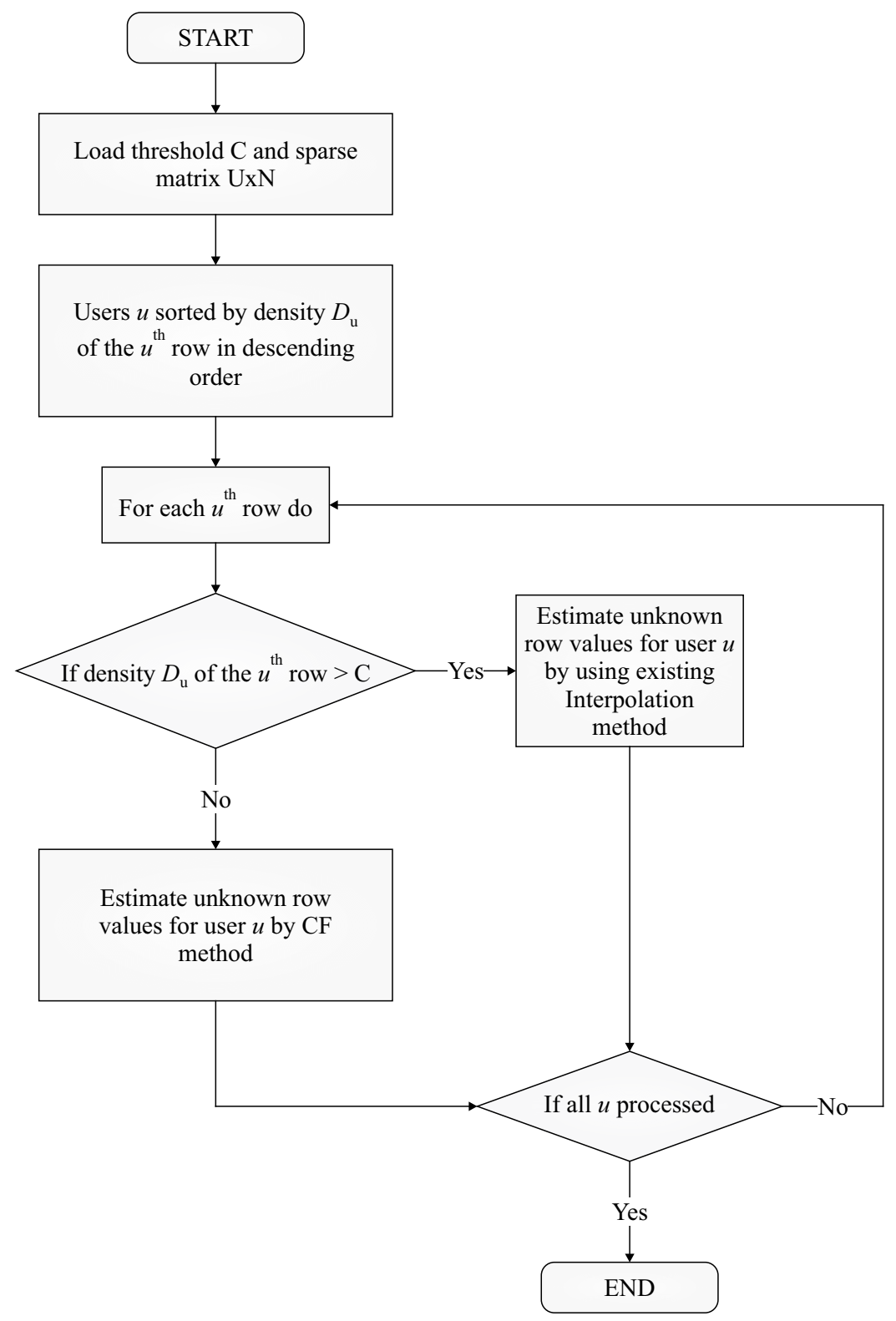

Fig. 4. Flow chart of the newly proposed channel estimation algorithm

\subsection{Evaluation metrics}

Root Mean Squared Error (RMSE) is a commonly used accuracy metric based on the absolute errors between the predictions and the actual values. It places greater weight on the higher errors which can have the greatest impact on the user decision. For this reason, RMSE should better reveal model performance differences than simple mean absolute error [31]. It is calculated using the following equation [12]

$$
R M S E=\sqrt{\frac{1}{K} \sum_{u, i}\left(p_{u, i}-r_{u, i}\right)^{2}},
$$

where $K$ is the total number of ratings over all users, and $p_{u, i}$ and $r_{u, i}$ denotes prediction rating and actual rating for user $u$ on item $i$.
In this paper, we are focused on the RMSE with the standard assumption that the errors are unbiased and follow a normal distribution.

\section{Proposed wireless channel estimation algorithm}

To address the challenges of wireless channel estimation on non-pilot subcarriers in OFDM systems, especially the sensitivity to the number and location of the pilot subcarriers, we propose the new channel estimation algorithm as shown in Fig. 4. The basic idea is to combine commonly used interpolation methods with memorybased $\mathrm{CF}$ techniques. 
The input data is the matrix $\mathbf{r}$ with dimensions $U \times N$, where $U$ is the number of users and $N$ the number of subcarriers. Let $r_{u, i}$ denote the frequency response value of user $u$ at subcarrier $i$. For each user $u$ in the $u^{\text {th }}$ row we have frequency response values of the channel at pilot subcarriers, while values at non-pilot subcarriers are unknown. Let $D_{u}$ denote the density of known values of $u^{\text {th }}$ matrix row. The output data will be a matrix filled with estimated values of the channel at non-pilot subcarriers. We assume that there are groups of users with very similar channel conditions and, consequently, similar channel frequency response values. This is the motivation to use collaborative filtering.

The proposed algorithm is realized in the following way. First, we sort the users in descending order based on their density of pilot subcarriers $D_{u}$ (known values). Depending on a constant $C$, which is a predefined threshold value of the pilot density for which interpolation should be performed, the algorithm will perform either existing interpolation method or CF method for a particular user (eg $5 \%$ ). The reasoning for such methodology is that interpolation methods work better when there is higher density of pilot subcarriers, otherwise they do not work well. A predefined value $C$ in the algorithm can be changed to find the best threshold value for which the prediction accuracy will be highest. Flow chart of channel estimation process is shown in Fig. 4.

In the first phase of the algorithm, for every user that satisfies $D_{u}>C$, we use existing interpolation method for estimating values of the channel at non-pilot subcarriers. Three existing interpolation methods will be considered in the simulations. In the second phase, the neighbourhood-based CF method is performed, consisting of the following steps for each user $u$ that meets the criterion $D_{u}<C$ :

(1) calculation of all correlation-based similarities $W_{x, u}$ for other users $x$ to the target user $u$ using Pearson correlations (4),

(2) identify subset of most similar users,

(3) for each unknown value at a non-pilot subcarrier $i$, calculate the estimation $r_{u, i}$ by taking the weighted average of values $r_{x, i}$ of all similar users $x$ at the same subcarrier using equation (5).

The CF method in each step considers all the values calculated in previous steps by interpolation method.

The algorithm's time complexity is dominated by the similarity calculations, which are performed in the overall time of $O\left(U^{\prime} U N\right)$, where $U^{\prime}$ is the number of "sparse" users for which $D_{u}<C$. The meaning of $O\left(U^{\prime} U N\right)$ is "of order of magnitude $U^{\prime}$ times $U$ times $N$ ", which is standard Big-O notation for the algorithm complexity. This is because the algorithm calculates the similarity of every such user $\left(U^{\prime}\right)$ and every other user $(U)$, and the complexity of calculating the Pearson correlation is proportional to the number of subcarriers: $O(N)$. In the best case, the number of users with the subcarrier density below the threshold $\left(D_{u}<C\right)$ is much less than $U$, so
$U \approx O(1)$ and the complexity becomes $O(U N)$. In the worst case, $U^{\prime} \approx U$ with the complexity of $O\left(U^{2} N\right)$.

\section{Simulation results and discussions}

In the experiments, we assumed pilot-aided comb type channel estimation based on irregular and scarce pilot arrangements. The main goals of simulations were:

- to compare RMSE for different selections of the algorithm threshold $C$,

- to compare existing interpolation methods and the newly proposed channel estimation algorithm, and

- to investigate the effect of the matrix density with different predefined algorithm thresholds on the proposed channel estimation algorithm performance.

In the simulation, we have used Rayleigh frequencyselective fading channel, which assumes complex Gaussian matrix elements with independent in-phase and quadrature components, modelled as the sum of $N$ Rayleigh flat fading sub-channels. We have used a Rayleigh fading model with power delay profile $P(t)=\left[1, e^{-1}, e^{-2}\right.$, $\left.e^{-3}, e^{-4}\right]$. The number of subcarriers per OFDM symbol was 128 , while the pilot patterns are non-equidistant with irregular pilot placement distribution. An irregular arrangement of pilot subcarriers for each user was chosen randomly in range for example from $5 \%$ to $30 \%$ (matrix density) without any pilot pattern structure in order to simulate the worst case scenario regarding performance and complexity of the system. In each experiment the algorithm was tested on 100 different channel matrices and the results were then averaged. The linear, second-order polynomial and cubic spline interpolation were simulated and compared with the proposed interpolation method using MATLAB built-in functions.

The first was to generate the whole matrix $r$ with frequency response values of the channel at all subcarriers. Then we inserted zeroes into the original matrix, forming a new matrix with some unknown values. Unknown values represent non-pilot subcarriers, while known values represent values of the channel at pilot subcarriers. Matrix density can be arbitrarily chosen in order to simulate different implementation scenarios. If not otherwise stated, in the experiments we generated 5 groups each having 20 users which have similar channel conditions and channel frequency response values inside the respective group.

At the end, after completion of proposed channel estimation algorithm RMSE metrics is used to evaluate the accuracy of the results obtained with proposed algorithm and finally to compare it with existing interpolation methods.

\subsection{Impact of threshold $C$ on proposed channel estima- tion algorithm}

In the first simulation scenario, on generated matrix with total average density $D=15 \%$ (The user densities $D_{u}$ was chosen randomly in range from $5 \%$ to $30 \%$.) we simulated the proposed algorithm in combination with 
RMSE

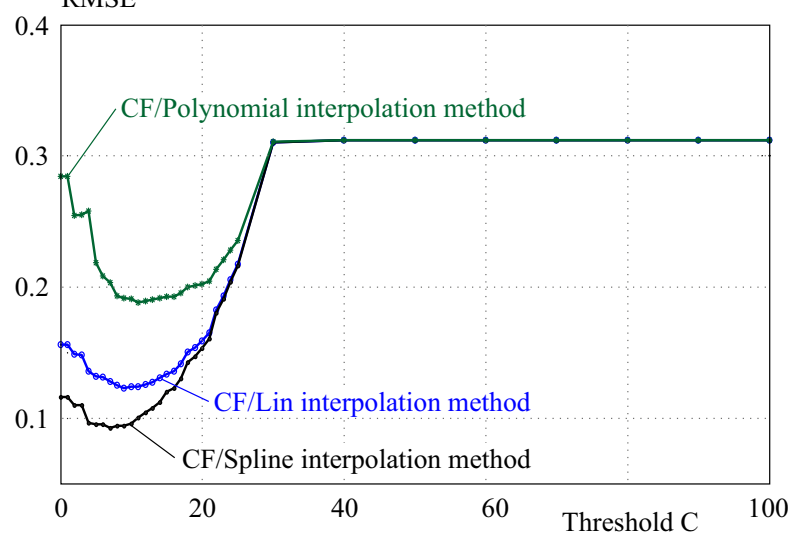

Fig. 5. RMSE vs threshold $C$ for proposed channel estimation algorithm

\section{RMSE}

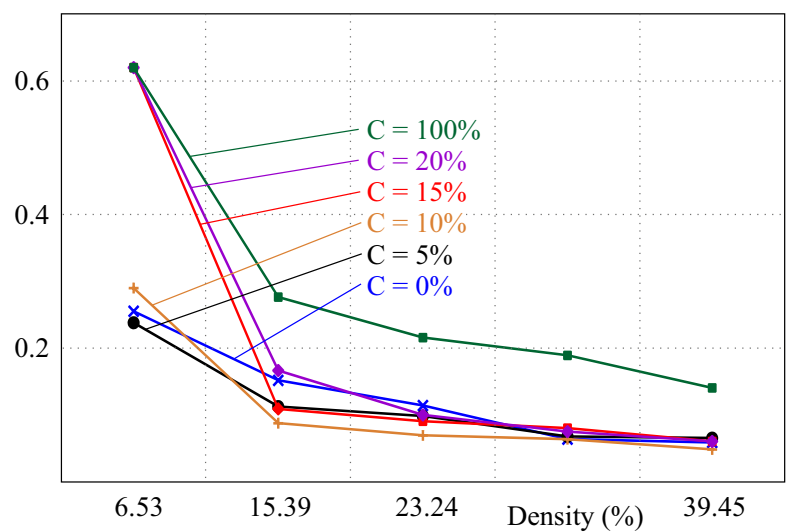

Fig. 7. RMSE vs total average matrix density for the proposed $\mathrm{CF} /$ Spline interpolation method (for different threshold values)

each of three different interpolation methods for different threshold values $C=[0$ to $25 \%]$ by $1 \%$ step and further, [30\% to $100 \%]$ by step of $10 \%$ as shown in Fig. 5 . The legends "CF/Lin", "CF/Spline", and "CF/Polynomial" denote the new channel estimation algorithm which combines the commonly used interpolation methods with the $\mathrm{CF}$ algorithm.

RMSE value at threshold $C=0 \%$ represents pure linear/spline/polynomial interpolation method, while RMSE value at threshold $C=100 \%$ represents pure $\mathrm{CF}$ method. From the results can be seen that among pure interpolation methods, best results are achieved by the spline interpolation method. Generally, it can be also seen that, in proposed channel estimation algorithm, comparing to the existing interpolation methods, better performance results are achieved for threshold values $C$ from $2 \%$ to $25 \%$ (depending on the proposed method). Gain is calculated using the following equation:

$$
\text { gain }=\frac{R M S E(C=0)-R M S E(C)}{R M S E(C=0)} \times 100 .
$$

For $\mathrm{CF} /$ Lin interpolation method, best RMSE is achieved for threshold $C=9 \%$, and comparing with

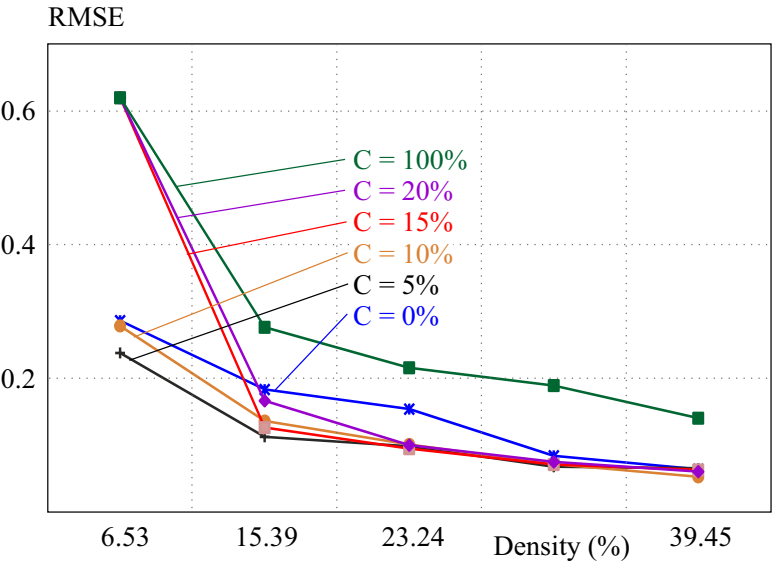

Fig. 6. RMSE vs total average matrix density for the proposed $\mathrm{CF} /$ Lin interpolation method (for different threshold values)

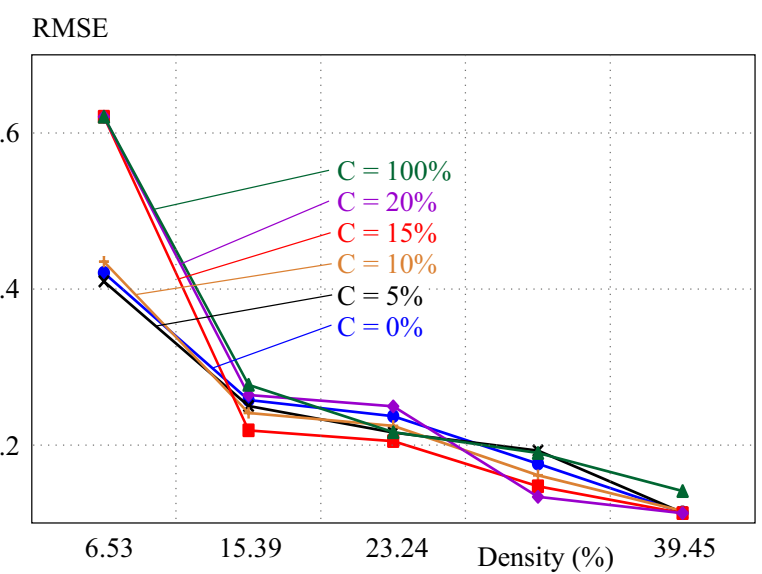

Fig. 8. RMSE vs total average matrix density for the proposed $\mathrm{CF} /$ polynomial interpolation method (for different threshold values)

RMSE for pure linear interpolation method $(C=0)$ the gain is $21.3 \%$. For CF/Spline and CF/Polynomial interpolation, best RMSE is achieved at thresholds $C=7 \%$ (gain of $20.32 \%$ ) and $C=11 \%$ (gain of $33.7 \%$ ), respectively.

\subsection{Impact of pilot subcarriers density on channel esti- mation algorithm}

In the next simulation scenario, five matrices $\boldsymbol{r}$ with different total average matrix densities in range from $6 \%$ to $40 \%$ were created in order to investigate the behaviour of RMSE values if density of pilot subcarriers is rising. Figures 6, 7, and 8 show the RMSE values with respect to the total average matrix density for each proposed interpolation method and different threshold values $C=$ [0\% $5 \% 10 \% 15 \% 20 \% 100 \%]$.

The results showed that performance of the proposed interpolation methods correlates with the density of the observed matrix. The result was expected since higher number of pilot subcarriers (higher average matrix density) in interpolation methods eventually gives better system performance results. 


$$
\operatorname{RMSE}(\mathrm{C}=5 \%)
$$

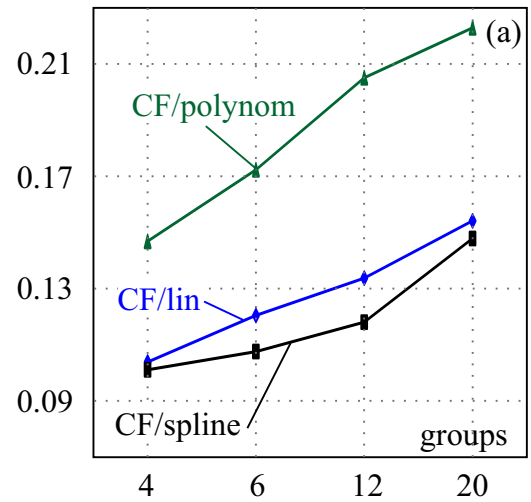

$\operatorname{RMSE}(\mathrm{C}=10 \%)$

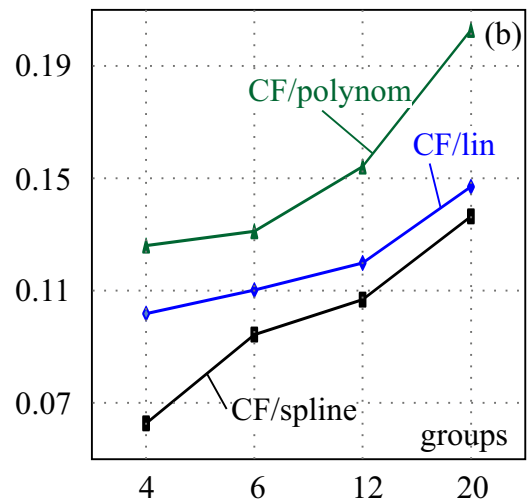

$\operatorname{RMSE}(\mathrm{C}=15 \%)$

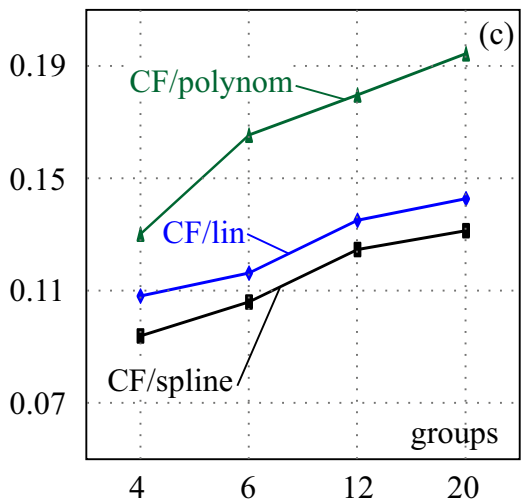

Fig. 9. RMSE vs number of groups of similar users in generated matrix for different thresholds: $(\mathrm{a})-C=5 \%,(\mathrm{~b})-C=10 \%$ and (c) $-C=15 \%$

It can also be observed that the proposed channel estimation algorithm mostly perform better on lower matrix densities, while on the higher densities their effect disappears. This is in line with the fact that CF techniques are less sensitive to the number of the pilot subcarriers.

\subsection{Impact of the user similarity amount on proposed channel estimation algorithm}

In the last simulation scenario, we generated matrices $\mathbf{r}$ with different total average matrix densities in range from $5 \%$ to $30 \%$ and differently grouped 120 users (4 groups with 30 users; 6 groups with 20 users; 12 groups with 10 users; 20 groups with 6 users) with similar frequency response values of the channel at pilot subcarriers. Figure 9 shows that RMSE is dependent on the number of similar user groups in the generated matrix: if the number of groups is high and thus the similarity between users is low, the proposed channel estimation algorithm is less accurate. On the other hand, when there is more similarity between users (fewer groups), the effect of CF techniques in the proposed channel estimation algorithm is more visible as predictions are getting more accurate.

The simulations showed that the proposed channel estimation algorithm improve prediction accuracy metrics, compared to usual channel estimation methods. Hence, they are more effective in situations when there are fewer pilot subcarriers. Also, as there were not assumed a regular pilot placement, the proposed channel estimation algorithm may be distributed in OFDMA systems with an irregular overall pilot arrangement.

\section{Conclusion}

High transmission rates and low BER in OFDM systems forces usage of some kind of interpolation methods for calculating the estimation of channel distortions at non-pilot subcarriers within the symbol transmissions. Those interpolation methods need to simultaneously fulfil low complexity and high accuracy, as they can have dramatical impact on the accuracy of channel estimation.
As efficiency of the channel estimation is a challenging problem in wireless systems with time and frequency selective variance, we proposed a new channel estimation algorithm which combines commonly used interpolation methods and a memory-based CF technique. The proposed interpolation channel estimation algorithm is less sensitive to the number and location of the pilot subcarriers. Compared to existing estimation methods, the simulation results of the proposed algorithm show improvements in prediction accuracy metrics for scenarios with low number of pilot subcarriers. However, by increasing the number of pilot subcarriers, the quality of the proposed channel estimation algorithm and, consequently, the accuracy of channel estimation decreases.

In conclusion, the proposed channel estimation algorithm based on collaborative filtering techniques for wireless channel estimation provides a practical algorithm for pilot-aided comb type OFDM based systems.

\section{REFERENCES}

[1] M. Zheng, Z. ZhengQuan, D. ZhiGuo, F. PingZhi, and L. HengChao, "Key Techniques for $5 \mathrm{G}$ Wireless Communications: Network Architecture, Physical Layer", Science China Information Sciences vol. 58, no. 4, pp. 1-20, 2015.

[2] S. Alam, J. W. Mark, and X. Shen, "Relay Selection Resource Allocation for Multi-User Cooperative OFDMA Networks", IEEE Transactions on Wireless Communications vol. 12, no. 5, pp. 2193-2205, 2013.

[3] J. G. Proakis, M. Salehi, "Digital Communications", fifth edition, McGraw-Hill, 2008.

[4] S. Adegbite, B. G. Stewart, and S. G. McMeekin, "Least Squares Interpolation Methods for LTE System Channel Estimation over Extended ITU Channels", International Journal of Information Electronics Engineering vol. 3, no. 4, pp. 414-418, 2013.

[5] S. Coleri, M. Ergen, A. Puri, and A. Bahai, "A Study of Channel Estimation in OFDM Systems", Proceedings of 56th Vehicular Technology Conference, Vancouver, Canada, vol. 2, pp. 894-898, 2002.

[6] Darshan, V. Adakane, and K. Vasudevan, "An Efficient Pilot Pattern Design for Channel Estimation in OFDM Systems", IEEE International Conference on Signal Processing Computing Control (ISPCC), Solan, India, September 2013. 
[7] A. Laraki, D. El Ouadghiri, and A. Jamali, "LTE Standard: Channel Estimation Algorithms from the Base Station to the Terminal", Journal of Theoretical \& Applied Information Technology vol. 68, no. 1, pp. 191-200, 2014.

[8] Y. Acar, H. Dogan, and E. Panayirci, "Pilot Symbol Aided Channel Estimation for Spatial Modulation-OFDM Systems its Performance Analysis with Different Types of Interpolations", Wireless Personal Communications vol. 94, no. 3, pp. 1387-1404, 2017.

[9] P. Fertl and G. Matz, "Channel Estimation in Wireless OFDM Systems with Irregular Pilot Distribution", IEEE Transactions on signal processing vol. 58, no. 6, pp. 3180-3194, 2010.

[10] L. Candillier, F. Meyer, and M. Boull, "Comparing State-of-theArt Collaborative Filtering Systems", Proceedings of the 5th international conference on Machine Learning Data Mining in Pattern Recognition Leipzig, Germany, pp. 548-562, July 2007.

[11] G. Linden, B. Smith, and J. York, "Amazon.com Recommendations: Item-To-Item Collaborative Filtering", IEEE Internet Computing vol. 7, no. 1, pp. 76-80, 2003.

[12] X. Su and T. M. Khoshgoftaar, "A Survey of Collaborative Filtering Techniques", Advances in Artificial Intelligence vol. 2009, no, 12, 2009.

[13] K. Fazel and S. Kaiser, "Multi-Carrier Spread Spectrum Systems: from OFDM MC-CDMA to LTE WiMAX", Second edition, John Wiley \& Sons Ltd, 2008.

[14] R. Hajizadeh, K. Mohamedpor and M. R. Tarihi, "Channel Estimation in OFDM System Based on the Linear Interpolation", Proceedings of 18th Telecommunications Forum TELFOR Belgrade, Serbia, 2010.

[15] S. Lee, H. Hung, and J. Wen, "A New Interpolation Method for Channel Estimation of OFDM Systems", Proceedings of TENCON IEEE Region 10 Conference, Nov-2005.

[16] R. Verma and S. Moghe, "A Survey on Pilot Symbol Assisted Channel Estimation in OFDM Systems", International Journal of Engineering Research \& Technology (IJERT) vol. 5, no. 8, pp. 316-320, 2016.

[17] P. Sure and C. M. Bhuma, "A Survey on OFDM Channel Estimation Techniques Based on Denoising Strategies", Engineering Science Technology an International Journal vol. 20, no. 2, pp. 629-636, 2017.

[18] S. Coleri, M. Ergen, A. Puri, and A. Bahai, "Channel Estimation Techniques Based on Pilot Arrangement in OFDM Systems", IEEE Transactions on broadcasting vol. 48, no. 3, pp. 223-229, 2002.

[19] M. K. Ozdemir and H. Arslan, "Channel Estimation for Wireless OFDM Systems", IEEE Communications Surveys \& Tutorials vol. 9, no. 2, pp. 18-48, 2007.

[20] M. Rana, J. Kim, and W. Cho, "Low Complexity Downlink Channel Estimation for LTE Systems", Proceedings of 12th International Conference on Advanced Communication Technology (ICACT) Phoenix Park, South Korea,, pp. 1198-1202, 2010.

[21] Y. Soo Cho, J. Kim, W. Yang and C. Kang, "MIMO-OFDM Wireless Communications with MATLAB", John Wiley \& Sons (Asia) Pte Ltd, 2010

[22] M. Hsieh and C. Wei, "Channel Estimation for OFDM Systems Based on Comb-Type Pilot Arrangement in Frequency Selective Fading Channels", IEEE Transactions on Consumer Electronics vol. 44, no. 1, pp. 217-225, 1998.

[23] M. Henkel, C. Schilling, and W. Schroer, "Comparison of Channel Estimation Methods for Pilot Aided OFDM Systems", Proceedings of IEEE 65th Vehicular Technology Conference (VTC) Dublin, Ireland, pp. 1440-1444, 2007

[24] X. Wang and K. J. R. Liu, "OFDM Channel Estimation Based on Time-Frequency Polynomial Model of Fading Multipath
Channel", Proceedings of IEEE 54th Vehicular Technology Conference (VTC) Atlantic City, USA, pp. 460-464, 2001.

25] S. G. Kang, Y. M. Ha, and E. K. Joo, "A Comparative Investigation on Channel Estimation Algorithms for OFDM in Mobile Communications", IEEE Transactions on broadcasting vol. 49, no. 2, pp. 142-149, 2003.

26] F. Cacheda, V. Carneiro, D. Fernandez, and V. Formoso, "Comparison of Collaborative Filtering Algorithms: Limitations of Current Techniques Proposals for Scalable", Journal Transactions on the Web (TWEB) vol 5, no, 1, 2011.

27] B. Sarwar, G. Karypis, J. Konstan, and J. Riedl, "Item-Based Collaborative Filtering Recommendation Algorithms", Proceedings of the 10th international conference on World Wide Web (WWW10) Hong Kong, pp. 285-295, 2001.

28] J. S. Breese, D. Heckerman, and C. Kadie, "Empirical Analysis of Predictive Algorithms for Collaborative Filtering", Proceedings of the 14th Conference on Uncertainty in Artificial Intelligence, Wisconsin, USA, pp. 43-52, 1998.

[29] S. Najafi and Z. Salame, "Evaluating Prediction Accuracy for Collaborative Filtering Algorithms in Recommender Systems", Student thesis Degree Project in Technology, Stockholm, 2016.

[30] P. Resnick, N. Iacovou, M. Suchak, P. Bergstrom, and J. Riedl, "Grouplens: an Open Architecture for Collaborative Filtering of Netnews", Proceedings of the ACM Conference on Computer Supported Cooperative Work, North Caroline, New York, USA, pp. 175-186, 1994

31] T. Chai and R. R. Draxler, "Root Mean Square Error (RMSE) or Mean Absolute Error (MAE)? - Arguments Against Avoiding RMSE in the Literature", Geoscietific Model Development vol. 7 , no. 3 , pp. $1247-1250,2014$

Received 25 March 2019

Velimir Švedek received a Masters Degree from the Faculty of Electrical Engineering and Computing at the University of Zagreb, at the Department of Telecommunications in 2004. He is pursuing a PhD in Telecommunications at the University of Zagreb, Faculty of Electrical Engineering and Computing. His research interests include optimization and resource allocation algorithms in OFDMA systems.

Adrian Satja Kurdija is a research assistant at the University of Zagreb, Faculty of Electrical Engineering and Computing, Consumer Computing Lab. He received a masters degree in Computer Science and Mathematics from the University of Zagreb in 2015. His $\mathrm{PhD}$ project is Recommender System for Service-Oriented Architecture. He has published in IEEE Communications Letters, Wireless Networks, European Journal of Operational Research, International Journal of Web and Grid Services, and Knowledge-based systems.

Željko Ilić received the $\mathrm{BSc}, \mathrm{MSc}$, and $\mathrm{PhD}$ degrees in electrical engineering from the Faculty of Electrical Engineering and Computing, University of Zagreb, Zagreb, Croatia, in 1995, 1999, and 2004, respectively. He is currently an Associate Professor in the Department of Telecommunications, Faculty of Electrical Engineering and Computing, University of Zagreb. His research interests include high-performance digital transmission, particularly to resource allocation algorithms for multiuser orthogonal frequency division multiplexing systems, medium access control protocols for broadband access networks and information theory. 